\title{
CONSTRAINTS ON THE TIME DELAY BETWEEN NUCLEOSYNTHESIS AND COSMIC-RAY ACCELERATION FROM OBSERVATIONS OF ${ }^{59} \mathrm{Ni}$ AND ${ }^{59} \mathrm{Co}$
}

\author{
M. E. Wiedenbeck, ${ }^{1}$ W. R. Binns, ${ }^{2}$ E. R. Christian, ${ }^{3}$ A. C. Cummings, ${ }^{4}$ B. L. Dougherty, ${ }^{1}$ P. L. Hink, ${ }^{2}$ \\ J. Klarmann, ${ }^{2}$ R. A. Leske, ${ }^{4}$ M. Lijowski, ${ }^{2}$ R. A. Mewaldt,${ }^{4}$ E. C. Stone, ${ }^{4}$ M. R. Thayer, ${ }^{4}$ \\ T. T. VON Rosenvinge, ${ }^{3}$ AND N. E. YANASAK ${ }^{1}$ \\ Received 1999 February 26; accepted 1999 July 15; published 1999 August 18
}

\begin{abstract}
Measurements of the abundances of cosmic-ray ${ }^{59} \mathrm{Ni}$ and ${ }^{59} \mathrm{Co}$ are reported from the Cosmic-Ray Isotope Spectrometer (CRIS) on the Advanced Composition Explorer. These nuclides form a parent-daughter pair in a radioactive decay which can occur only by electron capture. This decay cannot occur once the nuclei are accelerated to high energies and stripped of their electrons. The CRIS data indicate that the decay of ${ }^{59} \mathrm{Ni}$ to ${ }^{59} \mathrm{Co}$ has occurred, leading to the conclusion that a time longer than the $7.6 \times 10^{4} \mathrm{yr}$ half-life of ${ }^{59} \mathrm{Ni}$ elapsed before the particles were accelerated. Such long delays indicate the acceleration of old, stellar or interstellar material rather than fresh supernova ejecta. For cosmic-ray source material to have the composition of supernova ejecta would require that these ejecta not undergo significant mixing with normal interstellar gas before $\sim 10^{5} \mathrm{yr}$ has elapsed.
\end{abstract}

Subject headings: acceleration of particles — cosmic rays — nuclear reactions, nucleosynthesis, abundances supernovae: general

\section{INTRODUCTION}

A consensus has emerged that supernovae provide the power needed to maintain the observed energy density of cosmic rays in the Galaxy and that diffusive shock acceleration by supernova blast waves is the probable mechanism by which the particle acceleration occurs. Still controversial, however, is the nature of the population of seed particles that is accelerated. Among the sources that have been proposed for the seed population are (1) the outer layers of cool stars (Meyer 1985), (2) interstellar dust and gas (Meyer, Drury, \& Ellison 1997; Ellison, Drury, \& Meyer 1997), and (3) dust grains formed in the high-velocity ejecta from supernovae (Lingenfelter, Ramaty, \& Kozlovsky 1998). In the first two cases the material would be accelerated long after it was originally synthesized. In the third case, however, the nucleosynthesis and acceleration occur in the same supernova, and the time that elapses between these processes should be much shorter.

It was pointed out (Cassé \& Soutoul 1978; Soutoul, Cassé, \& Juliusson 1978) that radioactive nuclides that are produced in supernova explosions but can decay only by electron capture can be used to distinguish between models involving long and short time delays between nucleosynthesis and acceleration. In normal matter the electron capture decays proceed at a rate determined by the electron capture half-life, but once the nuclei are accelerated to high energies the orbital electrons are stripped off, making the particles effectively stable. Thus, if the acceleration occurred after a time delay short compared to the halflife, the parent nuclei should have survived. If the time delay was much longer than the half-life, the radioactive decays would have occurred, replacing the parent nuclei with their daughter products. It is possible to investigate a range of possible acceleration timescales by utilizing several electroncapture nuclides with different half-lives such as ${ }^{59} \mathrm{Ni}\left(T_{1 / 2}=\right.$ $\left.7.6 \times 10^{4} \mathrm{yr}\right)$ and ${ }^{57} \mathrm{Co}\left(T_{1 / 2}=0.74 \mathrm{yr}\right)$.

\footnotetext{
${ }^{1}$ Jet Propulsion Laboratory, California Institute of Technology, Pasadena, CA 91109

${ }^{2}$ Washington University, St. Louis, MO 63130.

${ }^{3}$ NASA/Goddard Space Flight Center, Greenbelt, MD 20771.

${ }^{4}$ California Institute of Technology, Pasadena, CA 91125.
}

Previous observations of the isotopes ${ }^{59} \mathrm{Ni}$ and ${ }^{59} \mathrm{Co}$ have been reported from experiments on ISEE 3 (Leske 1993), Ulysses (Connell \& Simpson 1997), and Voyager (Lukasiak et al. 1997; Webber 1997). Although limitations on statistical accuracy and mass resolution prevented these studies from definitively establishing the acceleration timescale, delays long enough to allow the decay of ${ }^{59} \mathrm{Ni}$ were generally favored.

We report new measurements of the abundances of ${ }^{59} \mathrm{Ni}$ and ${ }^{59} \mathrm{Co}$ from the Cosmic-Ray Isotope Spectrometer (CRIS) on the Advanced Composition Explorer (ACE) and discuss their implications for cosmic-ray acceleration.

\section{OBSERVATIONS}

The $A C E$ spacecraft, carrying a suite of high-resolution mass and charge spectrometers covering the energy range from $\sim 1 \mathrm{keV}$ nucleon ${ }^{-1}$ to $\sim 1 \mathrm{GeV}$ nucleon ${ }^{-1}$, was launched on 1997 August 25 and placed into a halo orbit about the L1 Lagrange point $1.5 \times 10^{6} \mathrm{~km}$ sunward of the Earth. The CRIS instrument measures cosmic-ray elemental and isotopic composition using the $d E / d x$ versus total energy technique. Energy losses and total energy are measured in four stacks of lithium-drifted silicon detectors, and particle trajectories are determined in a scintillating optical fiber trajectory hodoscope. CRIS has a large geometrical factor, $\sim 250 \mathrm{~cm}^{2} \mathrm{sr}$, which makes studies of rare cosmic-ray species possible. Details of the instrument design and performance have previously been reported (Stone et al. 1998).

The data used for this study were collected from 1997 August 28 through 1998 December 18, excluding several periods of significant solar energetic particle enhancements. Events were selected in which the incident particle penetrated at least the first two solid-state detectors in a stack and stopped in one of the following detectors. For these events, which fall in the energy range $\sim 170-500 \mathrm{MeV}$ nucleon $^{-1}$, two or more determinations of charge and mass were obtained and were required to be consistent to eliminate background events due to, for example, particles that underwent nuclear interactions in the instrument. Nuclei that stopped close to a dead layer in any of the $\mathrm{Si}(\mathrm{Li})$ detectors were rejected to avoid errors in the mass 

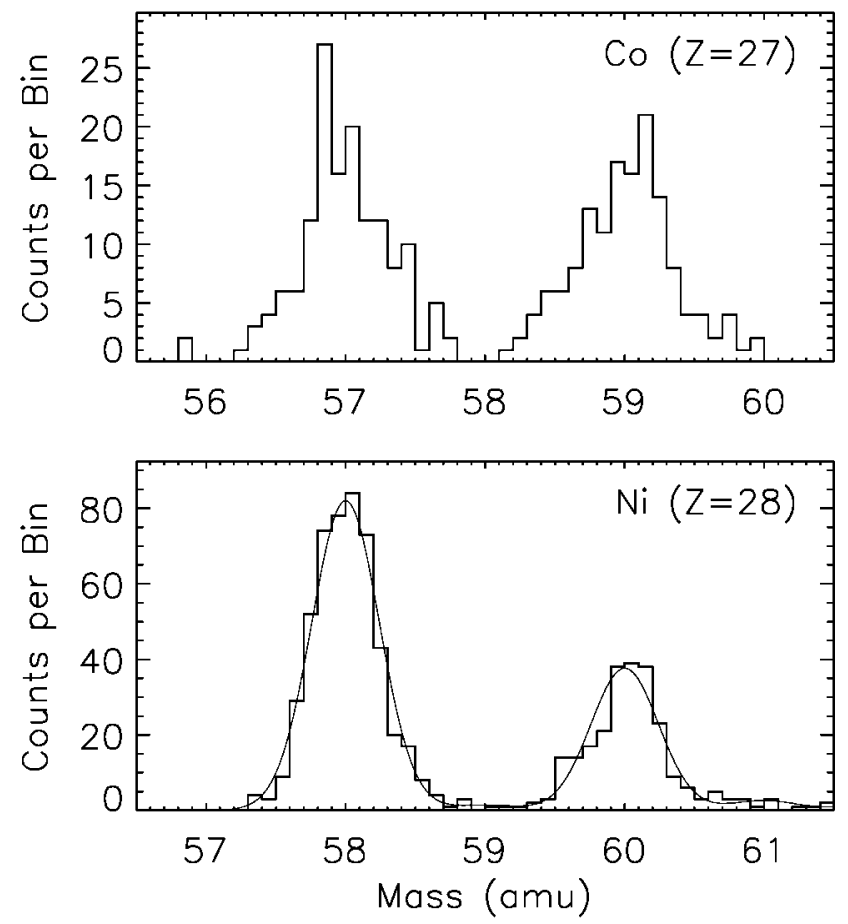

FIG. 1.-CRIS mass histograms. The Ni data (lower panel) were restricted to nuclei with angles of incidence within $20^{\circ}$ of the detector normal to obtain a high-resolution data set and minimize the contamination of the ${ }^{59} \mathrm{Ni}$ region with spillover from ${ }^{58} \mathrm{Ni}$ and ${ }^{60} \mathrm{Ni}$. The Co data (upper panel) had no angle restriction. The numbers of events and average mass resolution in these histograms are 293 and $0.33 \mathrm{amu}$ for Co and 785 and $0.25 \mathrm{amu}$ for Ni. The smooth curve in the lower panel shows the fitted distribution that was used in deriving the upper limit on the ${ }^{59} \mathrm{Ni}$ abundance.

determination related to incomplete collection of the ionization electrons. In addition, it was required that the three coordinate pairs measured along the particle trajectory lie on a straight line within the accuracy of the measurements.

Figure 1 shows the mass histograms that were obtained for Co and Ni. In order to reduce the spillover of ${ }^{58} \mathrm{Ni}$ and ${ }^{60} \mathrm{Ni}$ into the region of the lower abundance ${ }^{59} \mathrm{Ni}$ isotope, the $\mathrm{Ni}$ data have been restricted to angles of incidence less than $20^{\circ}$, taking advantage of the fact that the mass resolution is somewhat better at small angles. The $0^{\circ}-20^{\circ}$ data set contains approximately one-third of the $\mathrm{Ni}$ events available using the full angular acceptance of CRIS. For Co, with two isotopes of comparable abundance separated by two mass units, no angle cut was used.

To obtain the abundance of ${ }^{59} \mathrm{Ni}$, fits of the Ni mass distribution were performed using an empirical model for the observed peak shapes. This shape is nearly Gaussian when data are restricted to relatively small angles of incidence, as has been done in the $\mathrm{Ni}$ analysis. Identical peak widths were assumed for all of the $\mathrm{Ni}$ isotopes, and the separation between adjacent isotopes was derived from the ${ }^{58} \mathrm{Ni}$ and ${ }^{60} \mathrm{Ni}$ peaks. For Co the overlap of the mass peaks is negligible and the relative abundances can be simply obtained from the areas of the measured peaks. Small corrections were made for differences in the energy intervals over which the various isotopes were measured and for differences in the nuclear interaction losses in the instrument. Together these corrections to the isotope abundance ratios amounted to $\$ 3 \%$.

To relate abundances of Co isotopes to those of $\mathrm{Ni}$ isotopes, we performed a separate analysis using identical cuts for each
TABLE 1

Abundance Ratios at EARTh

\begin{tabular}{lcc}
\hline \hline Isotope Ratio & $\begin{array}{c}\text { ACE/CRIS Measured } \\
\text { Value }^{\mathrm{a}}\end{array}$ & $\begin{array}{c}\text { Calculated Secondary } \\
\text { Contribution }^{\mathrm{b}}\end{array}$ \\
\hline${ }^{59} \mathrm{Ni} /{ }^{60} \mathrm{Ni} \ldots \ldots$ & $<0.055$ & $0.049 \pm 0.012$ \\
${ }^{59} \mathrm{Co} /{ }^{60} \mathrm{Ni} \ldots \ldots$ & $0.221 \pm 0.021$ & $0.039 \pm 0.010$ \\
${ }^{57} \mathrm{Co} /{ }^{60} \mathrm{Ni} \ldots \ldots$ & $0.219 \pm 0.021$ & $0.208 \pm 0.021$ \\
\hline
\end{tabular}

${ }^{\text {a }}$ One standard deviation uncertainties.

${ }^{\mathrm{b}}$ Secondary contribution to numerator normalized to total ${ }^{60} \mathrm{Ni}$. See text for discussion of uncertainties.

element to obtain the $\mathrm{Co} / \mathrm{Ni}$ elemental abundance ratio. Energy spectra were produced for a wide range of elements, and these were fit using a common spectral form. Elemental abundances were derived from the normalization factors for these fits. In the measured charge distribution the Co peak is fully separated from the adjacent $\mathrm{Fe}$ and $\mathrm{Ni}$.

Table 1 lists the observed abundance ratios used in this study. The abundance ratio between the dominant $\mathrm{Ni}$ isotopes, ${ }^{60} \mathrm{Ni} /{ }^{58} \mathrm{Ni}$, is close to the solar system value. The abundances of the rare, stable isotopes ${ }^{61} \mathrm{Ni}$ through ${ }^{64} \mathrm{Ni}$ are not used in the present work but will be discussed in a separate publication.

\section{TRANSPORT CALCULATION}

A cosmic-ray transport calculation was performed to determine the fractions of the observed abundances attributable to secondary production by fragmentation of heavier nuclei during propagation in the Galaxy. The model and parameters were taken from Leske (1993) with the level of solar modulation adjusted to a value, $\phi=500 \mathrm{MV}$, appropriate to the time period of the CRIS measurements. The model successfully accounts for a sizable number of purely secondary isotopes in the subiron region and therefore should accurately predict the secondary contributions to ${ }^{59} \mathrm{Co}$ and ${ }^{59} \mathrm{Ni}$ if appropriate cross sections for producing these nuclides are used. The third column of Table 1 lists the calculated secondary contributions to the observed abundances.

The only significant secondary contributions to ${ }^{59} \mathrm{Ni}$ and ${ }^{59} \mathrm{Co}$ come from the fragmentation of ${ }^{60} \mathrm{Ni}$. Unfortunately, the relevant cross sections have not been measured. New estimates of the cross sections for the reactions ${ }^{60} \mathrm{Ni}(p, p n)^{59} \mathrm{Ni}$ and ${ }^{60} \mathrm{Ni}(p, 2 p){ }^{59} \mathrm{Co}$ were provided by W. R. Webber (1998, private communication). These values were obtained by extrapolating from recently measured cross sections for $(p, p n)$ and $(p, 2 p)$ fragmentation of the nuclides ${ }^{56} \mathrm{Fe}$ (Webber et al. 1998a) and ${ }^{52} \mathrm{Cr}$ (Webber et al. 1998b) which, like ${ }^{60} \mathrm{Ni}$, have four more neutrons than protons. At $600 \mathrm{MeV}$ nucleon ${ }^{-1}$ the estimated cross sections for producing ${ }^{59} \mathrm{Ni}$ and ${ }^{59} \mathrm{Co}$ are 68 and 40 mbarn, respectively. These are significantly less (factors of 1.5 and 1.25 , respectively) than the values previously obtained from the semiempirical formula of Webber, Kish, \& Schrier (1990), and they bring the calculated yield of secondary ${ }^{59} \mathrm{Ni}$ into reasonable agreement with the observed limit on the abundance of this nuclide (see below). The new cross sections are within $12 \%$ of values obtained from the semiempirical formulas of Silberberg, Tsao, \& Barghouty (1998).

We have taken the uncertainties on the calculated secondary contributions to ${ }^{59} \mathrm{Ni}$ and ${ }^{59} \mathrm{Co}$ to be $25 \%(1 \sigma)$, which is somewhat larger than the reported uncertainties in the relevant ${ }^{56} \mathrm{Fe}$ and ${ }^{52} \mathrm{Cr}$ cross section measurements to allow for additional uncertainty in the extrapolation to ${ }^{60} \mathrm{Ni}$.

As shown in Table 1, the observed limit on the abundance of ${ }^{59} \mathrm{Ni}$ is consistent with the expected secondary production 

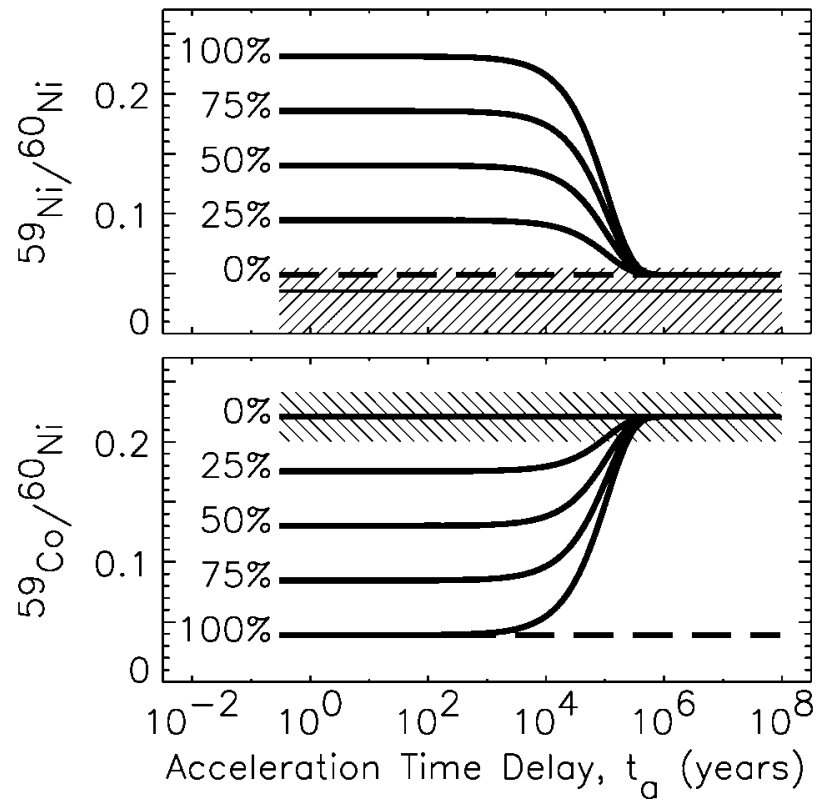

FIG. 2.-Calculated abundances at Earth of ${ }^{59} \mathrm{Ni}$ (upper panel) and ${ }^{59} \mathrm{Co}$ (lower panel) relative to ${ }^{60} \mathrm{Ni}$ are shown as a function of the time delay between nucleosynthesis and cosmic-ray acceleration. Calculated abundances are a combination of a secondary component (dashed lines) produced by nuclear fragmentation during transport and a surviving primary component. The total amount of primary mass number 59 material was obtained by subtracting the calculated ${ }^{59} \mathrm{Co}$ secondaries from the observed abundance of this isotope, since the observed ${ }^{59} \mathrm{Ni}$ is consistent with a purely secondary origin. The different curves correspond to different assumed fractional contributions of ${ }^{59} \mathrm{Ni}$ in the primary mass number 59 material, as indicated by the labels on the curves. The time dependences are the result of the exponential decay of the primary ${ }^{59} \mathrm{Ni}$ into ${ }^{59} \mathrm{Co}$ as the result of the electron-capture decay of ${ }^{59} \mathrm{Ni}$ before acceleration. The hatched regions indicate the abundances measured with CRIS, including $1 \sigma$ uncertainties. Although the fit yielded a finite ${ }^{59} \mathrm{Ni}$ abundance (thin line within the hatched region), the ${ }^{59} \mathrm{Ni}$ result is reported as an upper limit (see Table 1) because no ${ }^{59} \mathrm{Ni}$ peak is clearly discernible in the $\mathrm{Ni}$ histogram.

of this isotope. For ${ }^{59} \mathrm{Co}$ the measured value significantly exceeds the secondary contribution, and the difference of these quantities gives the abundance of primary ${ }^{59} \mathrm{Co}$ : $\left({ }^{59} \mathrm{Co}\right)_{\text {prim }} /{ }^{60} \mathrm{Ni}=0.182 \pm 0.021 \pm 0.010$. Here the first uncertainty is the measurement error; the second is the estimate of the uncertainty resulting from the calculated secondary correction. This ratio is consistent with the solar system value of 0.174 (Anders \& Grevesse 1989).

Another pure electron-capture nuclide that can be used for this type of study is ${ }^{57} \mathrm{Co}$, but it has a half-life $(0.74 \mathrm{yr})$ much shorter than that of ${ }^{59} \mathrm{Ni}$. The ${ }^{57} \mathrm{Co}$ abundance is consistent with a purely secondary origin (see Table 1), as expected if the time delay is longer than a few years. The calculation of the production of secondary ${ }^{57} \mathrm{Co}$ is relatively well constrained because measured cross sections are available for the reactions ${ }^{58} \mathrm{Ni}(p$, $2 p)^{57} \mathrm{Co}$ and ${ }^{58} \mathrm{Ni}(p, p n){ }^{57} \mathrm{Ni}$ (with the ${ }^{57} \mathrm{Ni}$ promptly decaying to ${ }^{57} \mathrm{Co}$ ), which are expected to account for more than threefourths of the production of secondary ${ }^{57} \mathrm{Co}$.

\section{DISCUSSION}

In a supernova explosion a variety of isobars of mass number 59 are produced. Those with $Z \leq 27$ promptly decay to ${ }^{59} \mathrm{Co}$, while those with $Z \geq 28$ decay to ${ }^{59} \mathrm{Ni}$. The ${ }^{59} \mathrm{Ni}$ can decay to ${ }^{59} \mathrm{Co}$ only by electron capture, and the half-life for this decay is long, $7.6 \times 10^{4} \mathrm{yr}$. Thus, the primary contribution to the

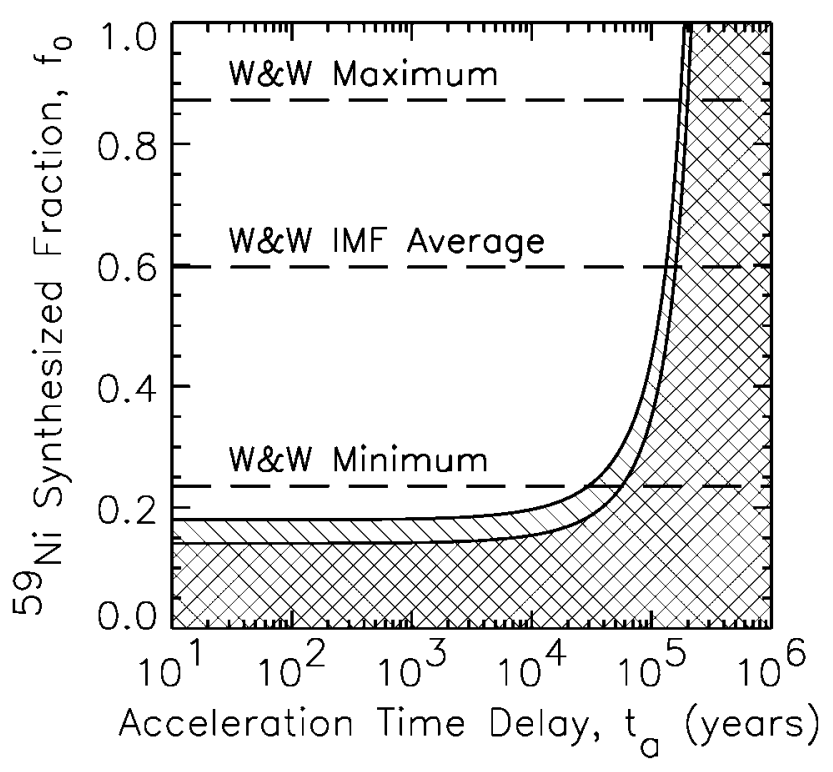

FIG. 3.-Combinations of $f_{0}$ and $t_{a}$ allowed by the CRIS data. Here $f_{0}$ is the fraction of primary mass number 59 material synthesized in the form of ${ }^{59} \mathrm{Ni}$ and $t_{a}$ is the time between nucleosynthesis and cosmic ray acceleration. The crosshatched region is a $98 \%$ confidence interval $(2 \sigma)$ derived taking into account only the uncertainties in the CRIS measurements. The hatched region is the result of also taking into account assumed uncertainties in the nuclear fragmentation cross sections ( $2 \sigma$ error of $50 \%$ ) added in quadrature with the abundance measurement errors. Dashed lines show values of the ${ }^{59} \mathrm{Ni}$ fraction obtained from a set of supernova models calculated by Woosley \& Weaver (1995), including the minimum and maximum values obtained for stars with masses between 11 and $25 M_{\odot}$, and an average obtained by weighting their results with a Salpeter initial mass function.

observed ${ }^{59} \mathrm{Co}$ could have been synthesized as a combination of ${ }^{59} \mathrm{Co}$ and ${ }^{59} \mathrm{Ni}$, with the latter isotope decaying before acceleration. The fraction $f\left(t_{a}\right)$ of the mass number 59 material which is in the form of ${ }^{59} \mathrm{Ni}$ at the time of acceleration $t_{a}$ is related to $f_{0}$, the fraction synthesized as ${ }^{59} \mathrm{Ni}$ (at $t=0$ ), by the equation

$$
f\left(t_{a}\right)=f_{0} \exp \left(-t_{a} / \tau\right),
$$

where $\tau \equiv T_{1 / 2} / \ln 2$ is the mean life for decay of ${ }^{59} \mathrm{Ni}$.

Figure 2 shows the abundances of ${ }^{59} \mathrm{Ni}$ and ${ }^{59} \mathrm{Co}$ that should be observed at Earth as a function of $t_{a}$ (the abscissa) and $f_{0}$ (the parameter distinguishing the different curves). These abundances contain both contributions due to secondaries produced during transport in the Galaxy (dashed lines, independent of $t_{a}$ ) and contributions due to surviving primaries which reflect the synthesized abundances of ${ }^{59} \mathrm{Ni}$ and ${ }^{59} \mathrm{Co}$ at short times and show the transformation to ${ }^{59} \mathrm{Co}$ for delays comparable to the ${ }^{59} \mathrm{Ni}$ half-life. The light line (obscured by the " $0 \%$ " curve in the case of ${ }^{59} \mathrm{Co}$ ) and hatched band in each panel indicate the abundance measurement obtained from CRIS with its $\pm 1 \sigma$ uncertainty. For ${ }^{59} \mathrm{Ni}$ the lower error bar has been extended to include a value of 0 because it is possible that spillover from ${ }^{58} \mathrm{Ni}$ and ${ }^{60} \mathrm{Ni}$ could be contributing the small number of events identified as ${ }^{59} \mathrm{Ni}$. Thus, for ${ }^{59} \mathrm{Ni}$ the upper bound of the shaded region represents an upper limit at the $84 \%$ confidence level.

Figure 3 shows the combinations of $f_{0}$ and $t_{a}$ that are consistent with the observed abundances. The cross-hatching indicates the region that is allowed, at the $98 \%$ confidence level, by the observed abundances with their associated measurement uncertainties. The inclusion of assumed 50\% uncertainties 
$(2 \sigma)$ in the calculated secondary corrections added in quadrature with the measurement uncertainties leads to the larger, hatched region.

Soutoul et al. (1978) assumed that the synthesized material should be predominantly ${ }^{59} \mathrm{Ni}$, as one would expect if all of the Fe-group nuclides were produced in nuclear statistical equilibrium with a neutron excess comparable to the value found in solar system material (0.002 excess neutrons per nucleon). However, recent detailed numerical models of the production of heavy nuclei in stars of various masses and metallicities and their subsequent ejection in supernova explosions (Woosley \& Weaver 1995) indicate that a variety of stellar zones and processes contribute to the Fe peak. In particular, Woosley \& Weaver (1995) find that for stars with solar metallicity and masses ranging from 11 to $25 M_{\odot}$, the fraction of ejected mass number 59 material in the form of ${ }^{59} \mathrm{Ni}$ can range from $\sim 24 \%$ to $\sim 87 \%$. The minimum, maximum, and average values of $f_{0}$ obtained from the Woosley \& Weaver (1995) models are indicated by dashed lines in Figure 3. The average value was obtained by weighting the models with a Salpeter initial mass function $\left(\propto m^{-2.35}\right.$, where $m$ is the mass of the star at the time of its formation), interpolating, and integrating over $11 M_{\odot} \leq m \leq$ $25 M_{\odot}$.

The model predictions for the production of ${ }^{59} \mathrm{Ni}$ overlap with the region allowed by the CRIS data only for time delays at least comparable to the ${ }^{59} \mathrm{Ni}$ half-life. The model with the lowest production of ${ }^{59} \mathrm{Ni}\left(m=18 M_{\odot}\right)$ nearly falls in the allowed region for short delay times; but only this one of the nine models calculated by Woosley \& Weaver (1995) has a ${ }^{59} \mathrm{Ni}$ fraction less than 0.29 , so this solution would require that cosmic rays originate from stars over a very narrow range of masses. Such a possibility-that cosmic-ray source material may have been synthesized under exceptional conditions where most of the mass number 59 material is produced in the form of ${ }^{59} \mathrm{Co}$ - can be investigated when one attempts to develop a consistent model to account for the synthesis of all the primary nuclides in the $\mathrm{Fe}-\mathrm{Ni}$ group. CRIS should be able to provide the observations needed for such a study.

A more plausible way to reconcile the CRIS observations with a short time delay between nucleosynthesis and acceleration is to hypothesize that the cross section for the reaction ${ }^{60} \mathrm{Ni}(p, p n){ }^{59} \mathrm{Ni}$ has been significantly overestimated. We regard this possibility as relatively unlikely since the cross section was extrapolated from measured cross sections of analogous reactions of neighboring nuclei. Nevertheless, direct measurements of cross sections for production of isotopes with mass numbers $57-59$ by fragmentation of ${ }^{60} \mathrm{Ni}$ are very important for unambiguously interpreting the cosmic-ray isotope observations.

The possibility remains that Fe-group nuclei could be promptly accelerated to an intermediate-energy $\lesssim 150 \mathrm{MeV}$ nucleon $^{-1}$, where they would be only partially stripped of their atomic electrons, with the remainder of the acceleration occurring on timescales $\gtrsim 10^{5} \mathrm{yr}$. This would allow primary ${ }^{59} \mathrm{Ni}$ to decay into ${ }^{59} \mathrm{Co}$ while preserving the pattern of the supernova abundances in the cosmic-ray source material. Such a scenario is difficult to rule out because the particles traverse only $\sim 1 \%$ of the total interstellar path length in $10^{5} \mathrm{yr}$, so alteration of abundances should be minimal except for electron-capture primaries.

Higdon, Lingenfelter, \& Ramaty (1998) have suggested that cosmic rays are accelerated in superbubbles formed by stellar winds and supernova explosions in OB associations. Since the ambient interstellar gas and dust should be rapidly blown out of superbubbles, Higdon et al. (1998) note that cosmic rays originating in such an environment can have the composition of supernova ejecta (except for primary electron capture nuclides), even though the time delay between nucleosynthesis and cosmic-ray acceleration must be significantly longer than the time to dissipate the energy from the explosion and thermalize the ejected material. In this scenario, ejecta from one supernova are accelerated by shocks from subsequent supernovae.

The CRIS data strongly indicate that a time $\gtrsim 10^{5}$ yr elapses between the synthesis of cosmic-ray source material and its acceleration to high energies. This timescale rules out models in which cosmic rays reach the energies at which they are observed as the result of a supernova accelerating its own ejecta. It is consistent with models in which the cosmic-ray seed population consists of old stellar or interstellar material or with models that are able to avoid mixing of supernova ejecta with ambient interstellar material for at least $\sim 10^{5} \mathrm{yr}$ before acceleration occurs.

We are grateful to the large group of dedicated individuals that contributed to the development of the CRIS instrument (listed in Stone et al. 1998). We thank W. R. Webber for providing new cross section estimates prior to publication. This research was supported by the National Aeronautics and Space Administration at the California Institute of Technology (under grant NAG5-6912), the Jet Propulsion Laboratory, the Goddard Space Flight Center, and Washington University and by the McDonnell Center for the Space Sciences at Washington University.

\section{REFERENCES}

Anders, E., \& Grevesse, N. 1989, Geochim. Cosmochim. Acta, 53, 197

Cassé, M., \& Soutoul, A. 1978, ApJ, 200, L75

Connell, J. J., \& Simpson, J. A. 1997, ApJ, 475, L61

Ellison, D. C., Drury, L. O’C., \& Meyer, J.-P. 1997, ApJ, 487, 197

Higdon, J. C., Lingenfelter, R. E., \& Ramaty, R. 1998, ApJ, 509, L33

Leske, R. A. 1993, ApJ, 405, 567

Lingenfelter, R. E., Ramaty, R., \& Kozlovsky, B. 1998, ApJ, 500, L153

Lukasiak, A., McDonald, F. B., Webber, W. R., \& Ferrando, P. 1997, Adv. Space Res., 19, 747

Meyer, J.-P. 1985, ApJS, 57, 173

Meyer, J.-P., Drury, L. O’C., \& Ellison, D. C. 1997, ApJ, 487, 182
Silberberg, R., Tsao, C. H., \& Barghouty, A. F. 1998, ApJ, 501, 911

Soutoul, A., Cassé, M., \& Juliusson, E. 1978, ApJ, 219, 753

Stone, E. C., et al. 1998, Space Sci. Rev., 86, 283

Webber, W. R. 1997, Space Sci. Rev., 81, 107

Webber, W. R., Kish, J. C., Rockstroh, J. M., Cassagnou, Y., Legrain, R., Soutoul, A., Testard, O., \& Tull, C. 1998a, ApJ, 508, 949

Webber, W. R., Kish, J. C., \& Schrier, D. A. 1990, Phys. Rev. C, 41, 566

Webber, W. R., Soutoul, A., Kish, J. C., Rockstroh, J. M., Cassagnou, Y., Legrain, R., \& Testard, O. 1998b, Phys. Rev. C, 58, 3539

Woosley, S. E., \& Weaver, T. A. 1995, ApJS, 101, 181 